Reprod. Nutr. Dévelop., 1982, 22 (4), 697-711.

\title{
Etude in vitro de l'activité motrice des deux couches musculaires de l'oviducte chez la Lapine
}

\author{
A. SACHY, J.-P. ROUSSEAU
}

Laboratoire de Neurophysiologie végétative

(LA $n^{\circ} 308$, CNRS), Bâtiment SN4,

Université de Lille I, 59655 Villeneuve d'Ascq Cedex.

Summary. In vitro study of the motor activity of two muscle layers in the rabbit oviduct.

Electrical and mechanical activities in the circular layer and the longitudinal peritoneal muscle were recorded in vitro in the oviducts of untreated and estrogen-treated ovariectomized rabbits. The duration and frequency of the bursts were not significantly changed in either muscle layer by the hormonal treatment. Estradiol increased the propagation of electrical activity in the isthmus and the amplitude of the contractions as well as the synchronization between the mechanical and electrical activities in the two muscle layers. Recordings of both electrical and mechanical activities were necessary to estimate the effects of the estrogen on the muscylar layers.

Removal of the longitudinal fiber bundles modifying the pattern of electrical and mechanical activities in the circular layer of the isthmus only increased the frequency of circular bursts and the frequency and amplitude of intraluminal pressure changes in both groups of animals. It is supposed that removal of the longitudinal fiber bundles could have damaged the inhibitory isthmic innervation of the circular layer.

The EMG activity of the longitudinal layer was generally independent from that of the circular layer in the oviducts of estrogen-treated rabbits. In 24 p. 100 of records which showed a synchronization of the electrical and mechanical activities of both muscle layers in the isthmus, the times between the contractions of the two muscle layers varied from 0.1 to $3.2 \mathrm{sec}$, suggesting that they were mechanically coupled.

\section{Introduction.}

Si chez la Lapine, le transit de l'œuf dans la trompe a été bien décrit (revue dans Pauerstein, 1979), les mécanismes responsables de sa migration restent mal connus. Dans l'ampoule, la propulsion de l'œuf serait assurée par les mouvements ciliaires (Halbert et al., 1976), par les contractions musculaires (Anand et Guha, 1978) ou par l'action conjointe de ces deux facteurs (Verdugo et al., 1980). Dans l'isthme, seules des contractions musculaires se produisant près de l'œuf seraient capables de produire un gradient de pression suffisant pour surmonter les forces de résistance à son déplacement (Chatkoff, 1975).

L'activité de la couche circulaire a pu être appréciée grâce aux techniques électromyographiques et I'on sait depuis les travaux de Talo et Brundin (1973) que les contractions de la couche longitudinale interfèrent avec celles de la 
couche circulaire. En réalité, la motricité complexe de l'oviducte intact résulte de la mise en jeu de ces deux couches (Ichijo, 1960 ; Coutinho et al., 1975), qui diffèrent par ailleurs dans leur sensibilité aux catécholamines (Lindblom et al., 1979) et aux prostaglandines (Lindblom et al., 1978).

Ces résultats nous ont incités à enregistrer l'activité des deux couches musculaires de l'oviducte par des techniques mécanographique et électromyographique. La couche circulaire de l'oviducte est flanquée de deux bandelettes externes constituées de fibres longitudinales et situées dans le prolongement des ligaments accessoires. Dans l'ampoule, dans les parties distale et médiane de l'isthme, les bandelettes longitudinales sont nettement séparées l'une de l'autre. Elles s'élargissent dans l'isthme proximal jusqu'à former une enveloppe externe continue d'épaisseur inégale (Greenwald, 1961). En travaillant in vitro, on peut recueillir séparément l'activité électrique des deux couches musculaires et procéder à des destructions locales ou complètes des bandelettes longitudinales. Comme la motricité tubaire est soumise à des influences hormonales, nous avons choisi deux situations expérimentales aisément reproductibles: les oviductes sont prélevés sur des lapines castrées traitées ou non par les œstrogènes. Nous essaierons dans chacune de ces situations d'apprécier les relations fonctionnelles entre les deux couches par deux approches méthodologiques. Dans la première, nous comparons l'activité de la couche circulaire dans l'oviducte intact et dans l'oviducte disséqué, afin d'établir si les structures longitudinales modifient l'activité de la couche circulaire. Dans la seconde, nous étudions les relations temporelles entre les activités des deux couches musculaires.

\section{Matériel et méthodes.}

Les résultats sont obtenus in vitro sur les oviductes de 38 lapines Fauve de Bourgogne, pesant de 1,5 à $2 \mathrm{~kg}$. Les animaux ovariectomisés sont séparés en deux groupes. Ceux du premier groupe $(n=13)$ ne subissent aucun traitement hormonal, ceux du second $(n=25)$ reçoivent une injection intramusculaire quotidienne de $20 \mu \mathrm{g}$ de benzoate d'œstradiol en solution huileuse, pendant 7 jours au-delà du huitième jour. L'oviducte est prélevé deux à trois semaines après la castration chez les lapines du premier groupe, 18 à $20 \mathrm{~h}$ après la dernière injection chez les lapines castrées et traitées. L'activité motrice est étudiée sur l'oviducte entier ou sur l'oviducte disséqué, privé de ses structures musculaires longitudinales.

Dans chaque expérience, les deux oviductes sont prélevés et examinés sous le microscope binoculaire (microscope opératoire Carl Zeiss). Celui qui montre la meilleure motricité est seul utilisé. II est fixé horizontalement dans une cuve de plexiglass contenant du liquide physiologique maintenu à $37{ }^{\circ} \mathrm{C}$, constamment renouvelé et oxygéné par un mélange gazeux à 95 p. $100 \mathrm{~d}^{\prime} \mathrm{O}_{2}$ et 5 p. 100 de $\mathrm{CO}_{2}$. Le liquide physiologique dont le $\mathrm{pH}$ est de 7,4 est composé de $\mathrm{NaCl}: 133,5$ $\mathrm{mM} ; \mathrm{KCl}: 4,5 \mathrm{mM} ; \mathrm{CaCl}_{2}: 1,9 \mathrm{mM} ; \mathrm{MgCl}_{2}: 0,05 \mathrm{mM} ; \mathrm{NaH}_{2} \mathrm{PO}_{4}: 1,4 \mathrm{mM}$; $\mathrm{NaHCO}_{3}: 16,3 \mathrm{mM}$; glucose : 7,8 mM. 
Lorsqu'on étudie l'oviducte intact, on enlève seulement le plus de tissu adipeux possible, sans léser les ligaments accessoires. Pour l'étude de la couche circulaire isolée, les ligaments accessoires et les bandelettes longitudinales sont disséqués soigneusement sous une loupe opératoire. Des contrôles histologiques ultérieurs confirment que les structures longitudinales ont été éliminées.

L'activité électrique est recueillie par une ou plusieurs électrodes à succion analogues dans leur principe à celles décrites par Talo et Csapo (1970). Ces électrodes sont constituées par un fil de cuivre émaillé de $0,14 \mathrm{~mm}$ de diamètre introduit dans un cathéter de polyéthylène dont l'extrémité est reliée à une trompe à vide. L'électrode indifférente consiste en une grilie d'argent plongée dans le liquide physiologique. Nous utilisons des amplificateurs Grass, type P 511, dont la constance de temps est de 0,1 à $0,3 \mathrm{~s}$.

Les variations de la pression endoluminale sont recueillies par un capteur de pression comprenant un électromanomètre Telco type M52 relié à une cellule Bell et Howell type 4.327.21. La trompe est perfusée sous un débit constant de $20 \mu \mathrm{l} / \mathrm{min}$ par du liquide physiologique, au moyen d'un cathéter inséré dans la jonction utéro-tubaire (JUT). Toute contraction de la couche circulaire induit une augmentation de la résistance à l'écoulement.

Les variations de tension sont enregistrées sur l'oviducte intact, au moyen d'un transducteur Grass de type FTO3 utilisé en montage isométrique. Elles traduisent les contractions de la couche longitudinale. Un fil de nylon attaché à l'extrémité libre de l'oviducte est relié par l'intermédiaire d'une poulie de renvoi au capteur fixé sur une potence à crémaillère ; les déplacements verticaux de la potence permettent de régler l'étirement de la préparation. Nous effectuons toutes les mesures de tension à la longueur de repos (Csapo et Goodall, 1954); elie est obtenue en étirant la trompe de façon intermittente pendant la période d'équilibre où l'organe isolé s'adapte aux conditions de survie. Lorsqu'une faible tension d'environ $0,5 \mathrm{~g}$ devient mesurable, on réduit lentement l'étirement pour ramener la tension à une valeur nulle.

Les fréquences, durées et amplitudes moyennes des contractions sont calculées à partir des tracés obtenus pendant les deux premières heures d'enregistrement. Les valeurs moyennes des paramètres de l'activité électromyographique (EMG) sont obtenues à partir d'au moins 4 séquences comprenant chacune de 30 à 150 phénomènes électriques, enregistrées dans les différentes régions de l'oviducte.

Les calculs de signification sont réalisés à l'aide du test non paramétrique de Wilcoxon.

\section{Résultats.}

I. - Caractéristiques de l'activité motrice de la couche circulaire dans l'oviducte intact.

L'activité EMG de la couche circulaire est recueillie par des électrodes placées entre les insertions des ligaments accessoires, même dans l'isthme proximal où la couche longitudinale entoure complètement l'oviducte. A ce niveau, l'acti- 

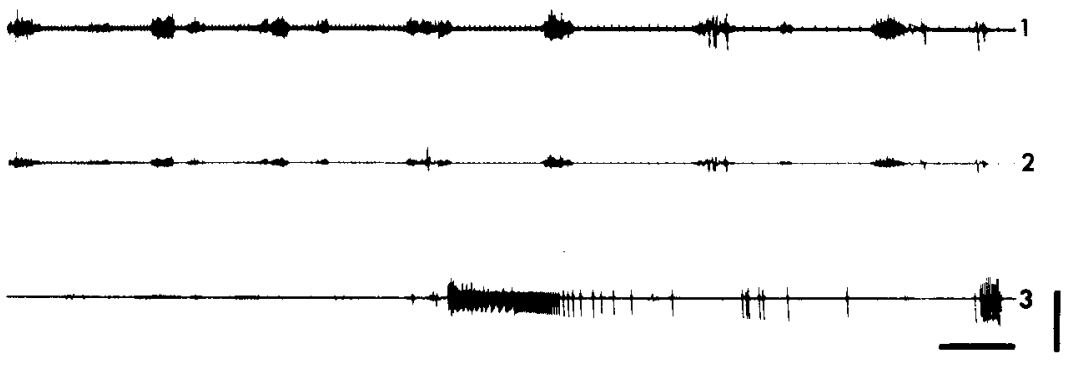

FIG. 1. - Enregistrements de l'activité électrique de l'isthme proximal sur un oviducte intact. L'activité EMG est recueillie simultanément : en un point de l'isthme proximal où la couche longitudinale est la plus mince (ligne 1) ; directement sur la couche circulaire dénudée par ablation ponctuelle de la couche longitudinale superficielle (ligne 2) ; sur la bandelette longitudinale (ligne 3). Les trois électrodes sont voisines, distantes de quelques millimètres. L'activité EMG recueillie sur la ligne 1 est identique à celle de la couche circulaire dénudée. La plus faible amplitude des phénomènes électriques observée à ce niveau (ligne 2) résulte vraisemblablement d'une lésion de la couche circulaire lors de la destruction de la couche longitudinale. Les caractéristiques de ces activités diffèrent entièrement de celles de I'activité EMG de la bandelette longitudinale (ligne 3).

Etalonnages : Amplitude : 0,5 mV; Temps : $10 \mathrm{~s}$.

vité électrique recueillie en surface est en effet différente de celle recueillie en regard, sur la bandelette longitudinale adjacente, à quelques millimètres de distance. Elle est identique à celle de la couche circulaire dénudée (fig. 1).

Chez l'animal castré, les différentes régions de l'oviducte intact extériorisent une activité EMG composée de potentiels isolés et de potentiels groupés en salves (tableau 1). Les durées et les fréquences moyennes des salves sont respectivement de $5,4 \mathrm{~s}$ et $3,8 / \mathrm{min}$ dans l'ampoule, de $6,1 \mathrm{~s}$ et $2,2 / \mathrm{min}$ dans l'isthme médian. L'activité électrique $n^{\prime}$ est pas propagée : deux électrodes voisines, distantes de $2 \mathrm{~mm}$, recueillent des activités EMG dont les caractéristiques sont différentes. L'activité mécanique se traduit par des augmentations de la pression intratubaire très irrégulières, comprises entre 0,5 et $11 \mathrm{~mm}$ de $\mathrm{Hg}$, sans relation temporelle avec les activités électriques.

Chez l'animal castré, traité par les oestrogènes, les salves prédominent dans l'ampoule et dans l'isthme intacts : elles représentent de 70 à 85 p. 100 des activités (tabl. 1). Leur durée moyenne, de $1,3 \mathrm{~s}$ dans l'ampoule atteint $3,2 \mathrm{~s}$ à la jonction isthme-ampoule (JIA) et 7,5 s dans I'isthme proximal. La fréquence des phénomènes électriques est d'environ $4 / \mathrm{min}$ à la JIA; elle est plus faible dans l'isthme médian et proximal. L'activité électrique est propagée ; on n'observe pas de relation entre l'activité électrique et l'activité mécanique dans l'ampoule. Dans l'isthme, 90 à 100 p. 100 des activités EMG coöncident avec une augmentation de la pression qui se traduit par une courbe de forme assez irrégulière (fig. $2 \mathrm{~A}$ ). La fréquence des pics de pression est en moyenne de $4,2 / \mathrm{min}$, leur amplitude de $2,6 \mathrm{~mm}$ de $\mathrm{Hg}$. Leur durée, en moyenne de $16,4 \mathrm{~s}$, peut atteindre près d'une minute. 
TABLEAU 1

Caractéristiques de l'activité EMG de la couche circulaire

A - Pourcentage de salves par rapport au total des activités recueillies

\begin{tabular}{cccccc}
\cline { 2 - 6 } & Ampoule & JIA & $\begin{array}{c}\text { Isthme } \\
\text { distal }\end{array}$ & $\begin{array}{c}\text { Isthme } \\
\text { médian }\end{array}$ & $\begin{array}{c}\text { Isthme } \\
\text { proximal } \\
+ \text { JUT }\end{array}$ \\
\hline$T$ & $\begin{array}{c}91,7 \\
(0-100)\end{array}$ & $\begin{array}{c}78,6 \\
(43,1-100)\end{array}$ & $\begin{array}{c}74,3 \\
(0-100)\end{array}$ & $\begin{array}{c}83,6 \\
(17,8-100)\end{array}$ & $\begin{array}{c}94,2 \\
(8,4-100)\end{array}$ \\
\hline OE & 82,5 & 70,6 & 70,4 & 73,8 & 85,0 \\
& $(63-100)$ & $(0-100)$ & $(0-100)$ & $(0-100)$ & $(0-100)$ \\
\hline
\end{tabular}

B - Durée des salves (en s)

\begin{tabular}{|c|c|c|c|c|c|}
\hline & Ampoule & JIA & $\begin{array}{l}\text { Isthme } \\
\text { distal }\end{array}$ & $\begin{array}{l}\text { Isthme } \\
\text { médian }\end{array}$ & $\begin{array}{c}\text { Isthme } \\
\text { proximal } \\
+ \text { JUT }\end{array}$ \\
\hline$T$ & $\begin{array}{c}5,4 \\
(0,5-51,5)\end{array}$ & $\begin{array}{c}3,3 \\
(0,6-21,3)\end{array}$ & $\begin{array}{c}2,7 \\
(0,8-10,7)\end{array}$ & $\begin{array}{c}6,1 \\
(0,5-18,4)\end{array}$ & $\begin{array}{c}5,3 \\
(0,4-28,0)\end{array}$ \\
\hline $\mathrm{OE}$ & $\begin{array}{c}1,3 \\
(0,5-8,4)\end{array}$ & $\begin{array}{c}3,2 \\
(1,0-10,7)\end{array}$ & $\begin{array}{c}3,3 \\
(1,8-6,0)\end{array}$ & $\begin{array}{c}5,3 \\
(0,8-19,6)\end{array}$ & $\begin{array}{c}7,5 \\
(0,8-62,8)\end{array}$ \\
\hline
\end{tabular}

C - Fréquences (par $\min$ )

\begin{tabular}{cccccc}
\cline { 2 - 5 } & Ampoule & JIA & $\begin{array}{c}\text { Isthme } \\
\text { distal }\end{array}$ & $\begin{array}{c}\text { Isthme } \\
\text { médian }\end{array}$ & $\begin{array}{c}\text { Isthme } \\
\text { proximal } \\
\text { + JUT }\end{array}$ \\
\hline $\mathrm{T}$ & $\begin{array}{c}3,8 \\
(1,2-7,5)\end{array}$ & $\begin{array}{c}2,6 \\
(0,7-9,7)\end{array}$ & $\begin{array}{c}1,9 \\
(1,0-8,2)\end{array}$ & $\begin{array}{c}2,2 \\
(0,3-3,6)\end{array}$ & $\begin{array}{c}1,1 \\
(0,6-3,9)\end{array}$ \\
\hline $\mathrm{OE}$ & $\begin{array}{c}4,5 \\
(1,3-7,3)\end{array}$ & $\begin{array}{c}3,9 \\
(0,8-7,2)\end{array}$ & $\begin{array}{c}4,1 \\
(3,0-6,9)\end{array}$ & $\begin{array}{c}2,4 \\
(0,7-6,2)\end{array}$ & $(0,8-2,9)$ \\
\hline
\end{tabular}

$T$ : lapines castrées : les résultats sont obtenus à partir d'enregistrements réalisés sur 7 oviductes. OE : lapines castrées, traitées par les cestrogènes : les résultats sont obtenus à partir d'enregistrements réalisés sur 12 oviductes.

JIA : jonction isthme-ampoule ; JUT : jonction utéro-tubaire.

Les nombres entre parenthèses indiquent les valeurs extrêmes rencontrées.

\section{II. - Activité motrice de la couche circulaire dans l'oviducte disséqué.}

Chez l'animal castré, l'ablation de la couche longitudinale entraîne deux modifications de l'électromyogramme : la partie proximale de l'isthme devient électriquement silencieuse et la fréquence des salves recueillies dans I'isthme médian augmente de 2,2 à $4,1 / \min (P=0,05)$. La pression intraluminale de base et les caractéristiques des pics de pression sont identiques, que l'oviducte soit intact ou disséqué. 


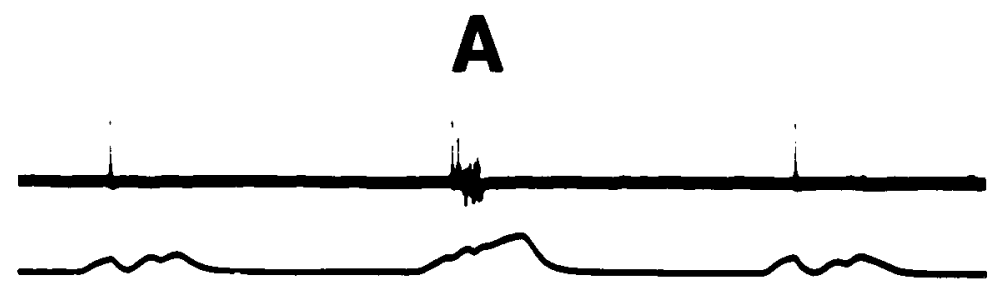

B

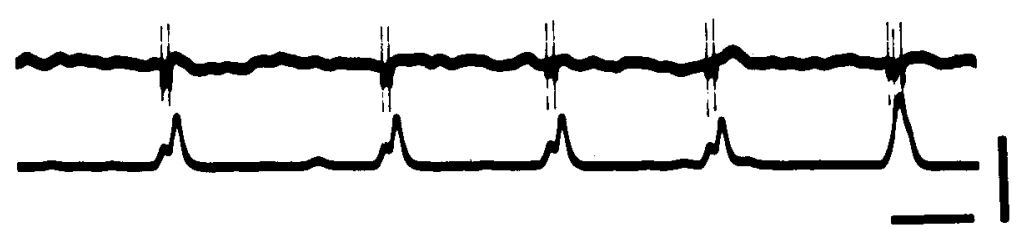

FIG. 2. - Activités électrique et mécanique de la couche musculaire circulaire recueillies sur un oviducte intact (en A) et sur un oviducte disséqué, après ablation des bandelettes longitudinales (en B). Les oviductes sont prélevés sur deux lapines castrées traitées par les œstrogènes.

Etalonnages : Amplitude : $1 \mathrm{mV}$ et $8,5 \mathrm{~mm}$ de $\mathrm{Hg}$; Temps : $10 \mathrm{~s}$.

Chez l'animal castré, traité par les œestrogènes, l'ablation des structures longitudinales modifie le mécanogramme (fig. $2 \mathrm{~B}$ ). Les variations de pression sont plus fréquentes sur la trompe disséquée que sur la trompe intacte $(4,2 / \mathrm{min}$ vs $3,2 / \mathrm{min} ; \mathrm{P}=0,05)$; leur durée est près de trois fois plus faible $(5,8 \mathrm{~s}$ vs $16,4 \mathrm{~s} ; \mathrm{P}<0,01)$, tandis que leur amplitude est doublée $(5,1 \mathrm{~mm}$ de $\mathrm{Hg}$ vs $2,6 \mathrm{~mm}$ de $\mathrm{Hg} ; P=0,02$ ). Leur durée, comme leur forme plus régulière, sont en relation avec le nombre et la fréquence des potentiels composant les salves recueillies de façon contemporaine (fig. $2 \mathrm{~B}$ ). En ce qui concerne l'activité électrique, l'absence de la couche longitudinale modifie seulement l'activité de la couche circulaire du segment proximal de l'isthme : la fréquence des salves de la couche circulaire y est significativement plus élevée $(P=0,05)$ dans l'oviducte disséqué $(3,5 / \mathrm{min})$ que dans l'oviducte intact $(1,4 / \mathrm{min})$. Dans l'ampoule et les autres parties de l'isthme, la fréquence des activités et la durée des salves ne sont pas altérées.

\section{III. - Enregistrements simultanés de l'activité des deux couches musculaires.}

$1^{\circ}$ Caractéristiques de l'activité motrice de la couche longitudinale.

a) Chez l'animal castré, l'activité EMG de la couche longitudinale, recueillie sur les différents segments de la trompe, apparaît sous la forme de salves de potentiels; les potentiels isolés représentent moins de 20 p. 100 des activités enregistrées (tabl. 2). Bien que les salves les plus longues soient recueillies à la JIA et dans l'ampoule, la durée moyenne des salves n'est pas significativement différente d'un segment de l'oviducte à l'autre. II en est de même pour les fréquences moyennes des activités électriques, qui sont de 1,3 à $2,8 / \mathrm{min}$. 


\section{TABLEAU 2}

Caractéristiques de l'activité EMG des bandelettes longitudinales

A - Pourcentage de salves par rapport au nombre total des activités recueillies

\begin{tabular}{|c|c|c|c|c|c|}
\hline & Ampoule & JIA & $\begin{array}{l}\text { Isthme } \\
\text { distal }\end{array}$ & $\begin{array}{l}\text { Isthme } \\
\text { médian }\end{array}$ & $\begin{array}{c}\text { Isthme } \\
\text { proximal } \\
+ \text { JUT }\end{array}$ \\
\hline $\mathbf{T}$ & $\begin{array}{l}79,1^{*} \\
(0-100)\end{array}$ & $\begin{array}{c}91,1 * \\
(77,8-100)\end{array}$ & $\begin{array}{c}81,2 \\
(25-100)\end{array}$ & 100 & $\begin{array}{c}81,0^{*} \\
(74,1-100)\end{array}$ \\
\hline $\mathrm{OE}$ & $100 *$ & $100^{*}$ & $\overline{(a)}$ & $\begin{array}{c}99,2 \\
(89-100)\end{array}$ & $\begin{array}{c}97,8^{*} \\
(67-100)\end{array}$ \\
\hline
\end{tabular}

* Différence significative : $\mathrm{P}<0,10$.

B - Durée des salves (en s)

\begin{tabular}{|c|c|c|c|c|c|}
\hline & Ampoule & JIA & $\begin{array}{l}\text { Isthme } \\
\text { distal }\end{array}$ & $\begin{array}{l}\text { Isthme } \\
\text { médian }\end{array}$ & $\begin{array}{l}\text { Isthme } \\
\text { proximal } \\
+ \text { JUT }\end{array}$ \\
\hline $\mathrm{T}$ & $\begin{array}{c}2,3 \\
(0,4-49,5)\end{array}$ & $\begin{array}{c}5,7 \\
(1,0-34,0)\end{array}$ & $\begin{array}{c}3,9 \\
(0,5-12,8)\end{array}$ & $\begin{array}{c}9,4 \\
(0,8-18,4)\end{array}$ & $\begin{array}{c}6,8 \\
(0,4-28,0)\end{array}$ \\
\hline $\mathrm{OE}$ & $\begin{array}{c}3,6 \\
(0,5-20)\end{array}$ & $\begin{array}{c}6,1 \\
(1,0-19,8)\end{array}$ & $\overline{\text { (a) }}$ & $\begin{array}{c}8,2 \\
(0,8-3,9)\end{array}$ & $\begin{array}{c}10,4 \\
(0,5-63,0)\end{array}$ \\
\hline
\end{tabular}

C - Fréquences (par $\mathrm{min}$ )

\begin{tabular}{cccccc}
\cline { 3 - 5 } & Ampoule & JIA & $\begin{array}{c}\text { Isthme } \\
\text { distal }\end{array}$ & $\begin{array}{c}\text { Isthme } \\
\text { médian }\end{array}$ & $\begin{array}{c}\text { Isthme } \\
\text { proximal } \\
+ \text { JUT }\end{array}$ \\
\hline$T$ & $\begin{array}{c}2,8 \\
(0,6-6,2)\end{array}$ & $\begin{array}{c}1,8 \\
(0,8-2,7)\end{array}$ & $\begin{array}{c}1,3 \\
(1,0-1,6)\end{array}$ & $\begin{array}{c}2,1 \\
(1,0-3,9)\end{array}$ & $\begin{array}{c}1,6 \\
(0,6-3,9)\end{array}$ \\
\hline OE & $\begin{array}{c}3,3 \\
(0,4-6,0)\end{array}$ & $\begin{array}{c}2,1 \\
(0,6-6,0)\end{array}$ & $\overline{(a)}$ & $(0,5-3,4)$ & $(0,3-3,4)$ \\
\hline
\end{tabular}

$T$ : lapines castrées : les résultats sont obtenus à partir d'enregistrements réalisés sur 5 oviductes. $\mathrm{OE}$ : lapines castrées, traitées par les œstrogènes : les résultats sont obtenus à partir d'enregistrements réalisés sur 8 oviductes.

JIA : jonction isthme-ampoule ; JUT : jonction utéro-tubaire.

Les nombres entre parenthèses indiquent les valeurs extrêmes rencontrées.

(a) : données insuffisantes.

Les contractions isométriques de l'oviducte et de ses ligaments accessoires se traduisent par de faibles augmentations de tension de $0,4 \mathrm{~g}$, qui durent en moyenne 12,9 s et apparaissent au rythme de 1,4/min. Dans l'ampoule, une activité EMG n'est pas toujours enregistrée en même temps qu'une variation de la tension. La relation est plus constante dans l'isthme, où 90 à 100 p. 100 des activités électriques apparaissent pendant une modification de la tension. Les délais 
entre les deux types d'activités varient cependant de 0,2 à 5,5 s et il n'existe pas de relation entre les caractéristiques de l'activité EMG et la durée ou l'amplitude du phénomène mécanique.

b) Chez l'animal castré, traité par les œstrogènes, l'amplitude moyenne des contractions est triplée par rapport à ce que l'on observe chez les lapines castrées, mais ni leur durée ni leur fréquence moyenne ne sont modifiées de façon significative. Il en est de même pour la durée et la fréquence moyenne des activités EMG ; par contre, les œstrogènes accroissent la proportion des salves par rapport à l'ensemble des activités électriques recueillies $(P<0,10)$ : les potentiels isolés sont extrêmement rares.

Dans la majorité des cas, on observe la propagation des activités EMG sous deux ou trois électrodes distantes d'un centimètre ou plus, ce qui n'est jamais observé chez la lapine castrée non traitée. Sous imprégnation œstrogénique, la vitesse apparente de conduction des salves, comprise entre 0,7 et $8 \mathrm{~cm} / \mathrm{s}$, est en moyenne de $1,6 \mathrm{~cm} / \mathrm{s}$. Le sens de propagation n'est pas fixe, il se modifie au cours d'un même enregistrement (fig. 3). De 90 à 100 p. 100 des activités EMG coïncident avec une augmentation de la tension. Plus la durée des salves est élevée, plus longue est la contraction. Lorsque les salves précèdent d'environ une seconde l'activité mécanique, la zone où elles sont enregistrées est la première activée, qu'il s'agisse de la JIA (fig. 3 A) ou de la JUT (fig. 3 B).
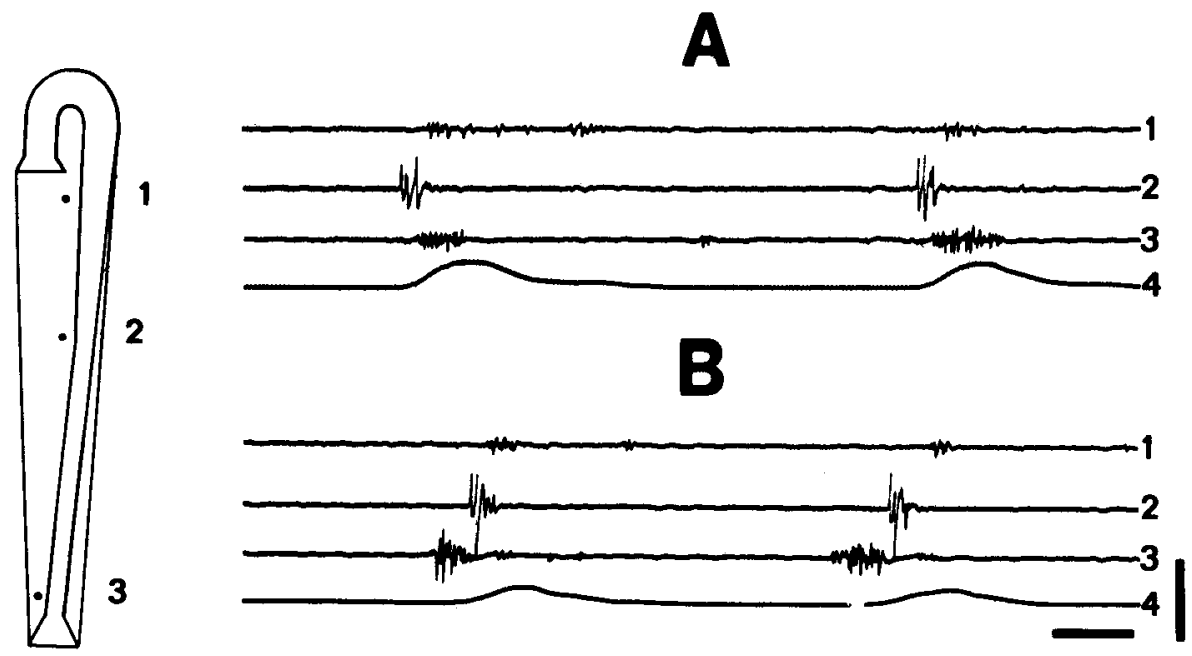

FIG. 3. - Lieux d'apparition de l'activité EMG propagée dans la couche musculaire longidutinale de l'oviducte intact, chez la Lapine castrée, traitée par les cestrogènes.

L'activité électromyographique est recueillie au niveau de l'ampoule (ligne 1), de la jonction isthme-ampoule (ligne 2) et de la jonction utéro-tubaire (ligne 3). La ligne 4 correspond à I'enregistrement des variations de tension.

En A, l'activité EMG apparaît à la jonction isthme-ampoule et se propage de façon divergente vers les extrémités ovarienne et utérine de l'oviducte. En B, les salves de potentiels apparaissent à la JUT puis remontent vers l'extrémité ovarienne de la trompe.

Etalonnages : Amplitude : $1 \mathrm{mV}, 6 \mathrm{~g}$; Temps : $2 \mathrm{~s}$. 
c) La comparaison des activités EMG des deux couches musculaires, recueillies sur des oviductes intacts, montre que les salves de la couche longitudinale sont plus longues que celles de la couche circulaire chez la Lapine castrée $(P<0,05)$. Sous imprégnation œestrogénique, les salves sont recueillies plus nombreuses dans la couche longitudinale que dans la couche circulaire $(P<0,01)$, ce qui $n^{\prime}$ est pas le cas chez les animaux castrés. Les fréquences des activités EMG sont semblables dans les deux couches, quel que soit l'état hormonal.

\section{$2^{\circ}$ Relations temporelles entre les activités motrices des deux couches.}

Chez la Lapine castrée non traitée, les variations de pression faibles et irrégulières, sont généralement indépendantes des variations de la tension. Nous recueillons l'activité électrique des deux couches musculaires, les variations de la pression intratubaire et celles de la tension dans 7 oviductes provenant de lapines castrées, traitées par les œstrogènes. Les quatre paramètres de l'activité motrice ont été analysés à partir de 21 séquences enregistrées en différents points de l'oviducte. La corrélation entre les deux phénomènes mécaniques est nulle ou faible pendant toute la durée de l'enregistrement dans 13 séquences sur 21. Deux électrodes voisines placées l'une sur la couche circulaire, l'autre sur une bandelette longitudinale recueillent alors des activités EMG asynchrones, de fréquence et d'aspect différents (fig. $4 \mathrm{~A}$ ) ; l'augmentation de la pression coïncide
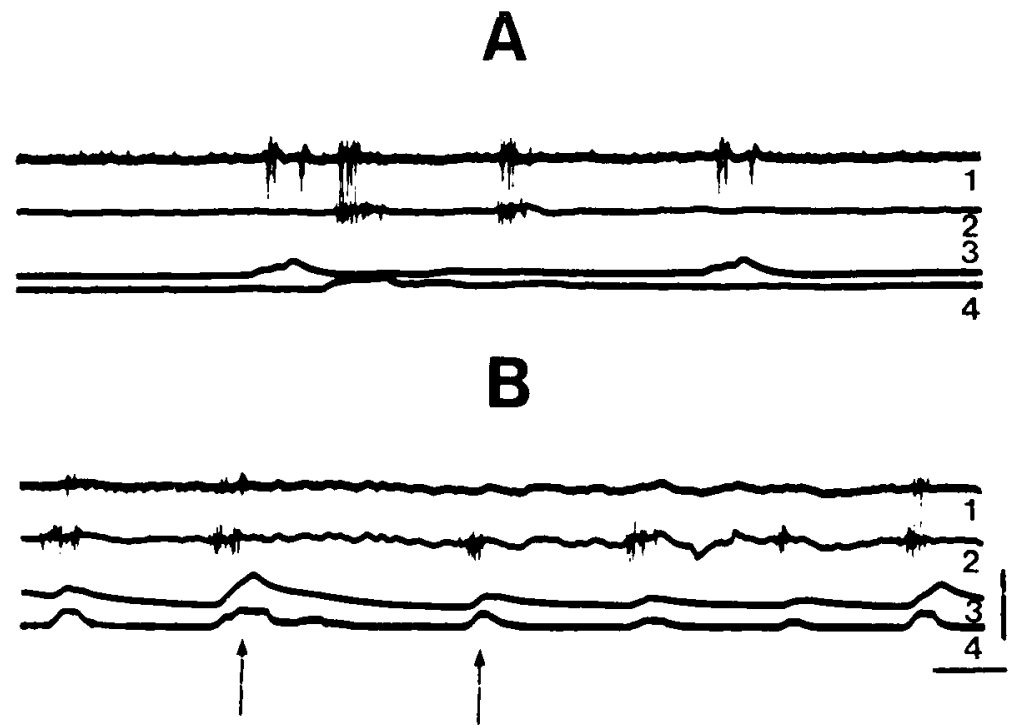

FIG. 4. - Relations entre l'activité de la couche circulaire et celle de la couche longitudinale chez la Lapine castrée, traitée par les cestrogènes.

On recueille l'EMG de la couche circulaire en 1, l'EMG de la couche longidutinale en 2, les variations de la pression intratubaire en 3 , les variations de la tension en 4 . En A, les activités mécaniques des deux couches sont indépendantes. En B, toutes les variations de pression correspondent à une augmentation de la tension ; la variation de pression est d'autant plus importante que l'augmentation de la tension contemporaine est grande (flèches). On note qu'en A les salves de la couche circulaire sont plus fréquentes que celles de la couche longitudinale ; l'inverse est observé en B.

Etalonnages : Amplitude : $0,5 \mathrm{mV}$ en $\mathrm{A} ; 1 \mathrm{mV}$ en $\mathrm{B} ; 8,5 \mathrm{~mm}$ de $\mathrm{HG} ; 3 \mathrm{~g} ;$ Temps : $10 \mathrm{~s}$. 
avec une salve de la seule couche circulaire (fig. $5 \mathrm{~A}$ ) ou l'augmentation de tension avec une activation de la couche longitudinale. Dans 8 séquences, les variations de pression coïncident avec une augmentation de la tension (fig. 4 B). Les deux phénomènes sont liés; l'augmentation de la pression est d'autant plus importante que la variation de tension est plus grande. Deux cas sont observés : i) Dans 3 de ces 8 séquences, la couche circulaire est électriquement inactive et une salve recueillie sur la couche longitudinale précède une augmentation de pression, contemporaine d'une variation de la tension (fig. 5 B). La contraction des fibres longidutinales affecte donc la pression intratubaire en l'absence d'une activation de la couche circulaire à l'endroit interrogé.
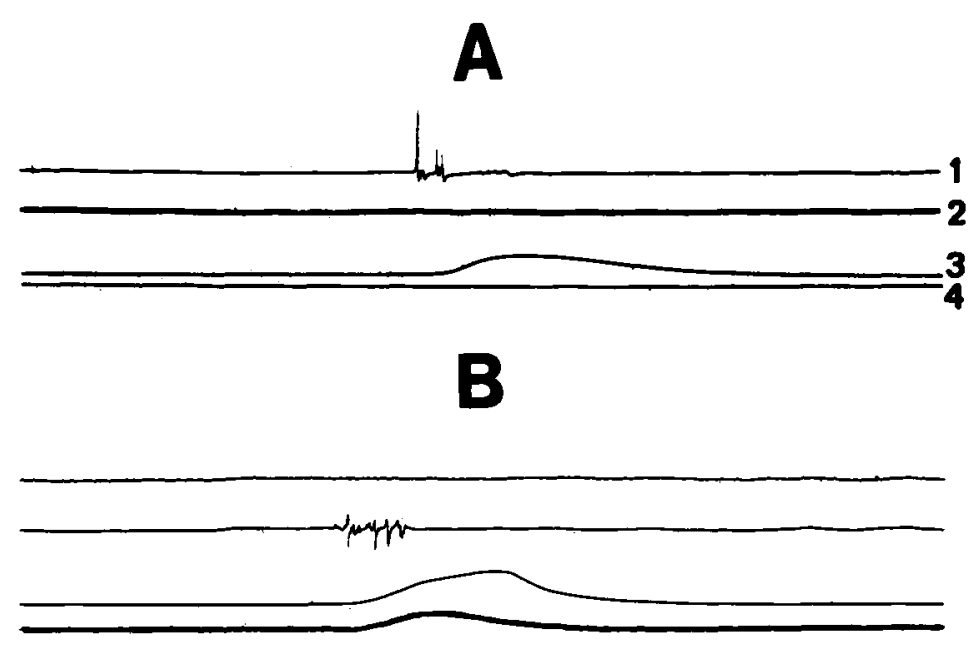

C

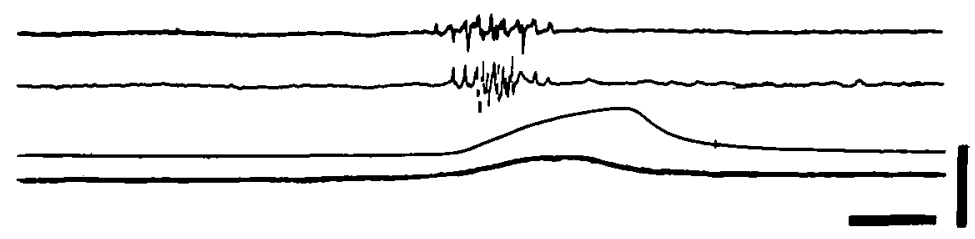

FIG. 5. - Relations entre les activités électrique et mécanique recueillies dans l'isthme intact, chez une lapine castrée traitée par les ostrogènes.

On enregistre en 1 l'activité EMG de la couche musculaire circulaire; en 2 celle de la couche musculaire longitudinale; en 3 les variations de la pression endoluminale et en 4 celles de la tension. En A, l'activité électrique de la couche circulaire précède l'augmentation de la pression. Aucune activité électrique de la couche longitudinale ni variation de la tension ne sont enregistrées. En B, l'activité EMG n'apparaît qu'au niveau de la couche longitudinale. La variation de tension coïncide cependant avec une augmentation de la pression endoluminale. En C, les deux couches musculaires sont simultanément actives. Les activités EMG correspondent à des variations de la pression endoluminale et de la tension.

Etalonnages : Amplitude : $1 \mathrm{mV}$ en $A ; 2 \mathrm{mV}$ en $B ; 0,5 \mathrm{mV}$ en $\mathrm{C} ; 17 \mathrm{~mm}$ de $\mathrm{Hg}$ en $\mathrm{A}$ et $\mathrm{B}$; $8,5 \mathrm{~mm}$ de $\mathrm{Hg}$ en $\mathrm{C} ; 6 \mathrm{~g}$; Temps : $2 \mathrm{~s}$. 
ii) Dans les 5 autres séquences, on recueille des activités EMG contemporaines dans les deux couches musculaires sous la forme de salves dont les caractéristiques de durée et de fréquence sont comparables (fig. $5 \mathrm{C}$ ). De telles activités sont observées dans l'ensemble de l'isthme, mais principalement dans sa partie proximale et à la JUT ( 3 séquences sur 5 ). Leur relation temporelle peut s'inverser d'une salve à l'autre au cours d'un même enregistrement : les salves de la couche circulaire précèdent ou suivent celles de la couche longitudinale de 0,1 à 3,2 s.

\section{Discussion.}

$1^{\circ}$ Remarques sur les activités EMG recueillies.

Nous observons sous imprégnation œstrogénique une relation étroite entre I'activité EMG de chaque couche musculaire et son activité mécanique. Les activités EMG se propagent sur toute la longueur de l'isthme, tant au niveau de la couche circulaire que des bandelettes longitudinales. Or, les œstrogènes assurent la synchronisation des activités élémentaires des fibres musculaires et favorisent la conduction musculo-musculaire de l'activité électrique dans la couche circulaire de I'oviducte (Talo et Brundin, 1970). L'activité électrique des bandelettes longitudinales est propagée le jour de la parturition, alors qu'en fin de gestation, la propagation des salves est lente et limitée (Talo et brundin, 1973). Or on sait que l'œstradiol prédomine le jour de la parturition, la sécrétion de progestérone étant alors retournée à son niveau de base (Challis et al., 1973, Harrington et Rothermel, 1977).

En ce qui concerne la couche longitudinale, Meiri et al. (1978) ne relèvent aucune différence dans l'aspect de l'activité EMG, que les oviductes soient prélevés sur des lapines castrées, des lapines en cestrus ou traitées par les œstrogènes. Dans nos expériences, ni la fréquence, ni la durée des salves recueillies sur les bandelettes longitudinales ne sont modifiées par le traitement hormonal. Les œstrogènes ne sont pourtant pas sans effet : outre que l'activité EMG se propage sur toute la longueur des bandelettes longitudinales, ce qui n'est pas le cas chez la Lapine castrée, ils accroissent la proportion des salves par rapport à l'ensemble des activités : les potentiels isolés deviennent extrêmement rares. L'amplitude des contractions est triplée et les relations entre activités électrique et mécanique sont plus étroites que chez la Lapine castrée. Ces résultats montrent que l'enregistrement de la seule activité EMG n'est pas suffisant pour apprécier l'effet des hormones stéroïdes.

Nous n'observons jamais de salves de 1,5 à 3,5 min de durée, comme en enregistrent chez la Lapine in vivo, Ruckebusch (1975) sur la couche circulaire, et Gonzalez de Vargas et al. (1976) sur les bandelettes longitudinales. La section de la JUT entraîne une forte réduction de l'activité EMG tubaire (Ruckebusch, 1975). II est possible que l'apparition de ces longues bouffées d'activité soit liée à l'intégrité des relations anatomo-fonctionnelles entre l'oviducte et l'utérus. On peut supposer que dans les conditions in vitro, l'absence de ces salves résulte de I'interruption de ces relations. Le fait que, comme Talo et Brundin (1973), Daniel et al. (1975), Meiri et al. (1978), nous ne recueillons pas in vitro ces types d'activité, vient à l'appui de cette hypothèse. 
$2^{\circ}$ Causes possibles des modifications observées dans l'activité motrice de la couche circulaire, sur la trompe disséquée.

Chez la Lapine castrée, la couche circulaire de l'isthme proximal devient électriquement silencieuse, lorsque les bandelettes longitudinales de l'oviducte sont enlevées. A ce niveau, celles-ci sont épaisses et leur dissection doit être plus poussée que dans le reste de l'isthme; la couche circulaire étant de faible épaisseur, la disparition de l'activité électrique est vraisemblablement due à sa lésion. On observe par contre, après l'ablation des structures longitudinales, une augmentation significative de la fréquence des salves, dans l'isthme médian chez l'animal castré, dans l'isthme proximal chez la Lapine castrée traitée par les œstrogènes. Sous imprégnation cestrogénique, la fréquence et l'amplitude des variations de la pression intratubaire sont plus élevées sur l'oviducte disséqué que sur l'oviducte intact. Or les augmentations de pression traduisent l'activité du seul isthme, voire même de sa partie proximale (Brundin, 1965 ; Talo, 1975). Tout se passe comme si la présence de la couche longitudinale limitait l'activité de la couche circulaire.

Si l'on tient compte de l'innervation intrinsèque de l'oviducte, on peut penser que la destruction d'une partie des neurones adrénergiques pourrait expliquer les différences observées entre l'activité de l'isthme disséqué et celle de l'isthme intact. La couche circulaire de l'isthme est richement innervée par le système de neurones adrénergiques à axones courts, dont les corps cellulaires sont situés dans le ganglion paracervical de Frankenhaüser : une fraction importante de ces axones suit le trajet des vaisseaux sanguins, qui disparaissent lors de la dissection des bandelettes longitudinales (revue dans Paton et al., 1978). On sait que la charge en noradrénaline est augmentée chez la Lapine sous œstrogène (Owman et Sjöberg, 1966). Or chez la Lapine sous imprégnation œstrogénique, la noradrénaline agit par l'intermédiaire des récepteurs $\alpha$-adrénergiques excitateurs, qui prédominent dans la couche circulaire de l'oviducte (Paton et al., 1978). Dans une telle hypothèse, l'ablation des structures longitudinales, détruisant l'innervation intrinsèque, devrait se traduire par une diminution de l'activité de la couche circulaire, plus prononcée chez la Lapine sous œestrogène que chez la Lapine castrée. C'est le phénomène inverse qui est observé. Une explication peut être avancée, en fonction de la récente découverte de terminaisons riches en Vasoactive Intestinal Peptide (VIP), particulièrement denses dans les musculeuses de I'isthme. Des cellules à VIP sont présentes dans le ganglion paracervical (Alm et al., 1980). L'activité contractile de la couche circulaire de l'isthme est fortement inhibée par l'administration de VIP (Walles et al., 1980). La fréquence plus élevée des contractions de la couche circulaire de l'isthme sur la trompe disséquée pourrait résulter de la destruction totale ou partielle de ce système de nerfs à fonction inhibitrice.

\section{$3^{\circ}$ Relations fonctionnelles entre les deux couches musculaires.}

L'activité des structures longitudinales est généralement indépendante de celle de la couche circulaire : les variations de la tension longitudinale et celles de la pression intratubaire ne sont pas simultanées ; de fréquence et d'aspect différents, les salves recueillies sur les deux couches sont nettement asynchrones: 
on enregistre une activité EMG sur l'une des couches, alors que l'autre reste au même instant silencieuse au point interrogé. Chaque couche musculaire possède son autonomie fonctionnelle, comme le suggèrent déjà les résultats de Talo et Brundin (1973).

Deux cas particuliers sont cependant rencontrés. Une salve de la couche longitudinale, une augmentation de la tension et de la pression intratubaire sont recueillies en même temps. La contraction des structures longitudinales modifie la pression endoluminale en l'absence de toute activation de la couche circulaire. Des résultats semblables sont obtenus in vitro par Talo et Brundin (1973) et in vivo par Gonzalez de Vargas et al. (1976). II est possible que l'action des fibres longitudinales s'explique par la structure particulière de l'isthme qui forme plusieurs anses sous-tendues par les ligaments accessoires et bordées latéralement par les bandelettes longitudinales. La contraction de ces structures aboutit probablement à resserrer les anses, ce qui obstrue plus ou moins complètement la lumière de l'oviducte et agit sur la pression intratubaire. Un tel mécanisme existe chez la Rate où les anses de l'oviducte sont encore plus marquées que chez la Lapine (Brundin et al., 1969).

Des activités EMG sont recueillies de façon contemporaine dans les deux couches musculaires, principalement dans la partie proximale de l'isthme et à la JUT. Un couplage fonctionnel des deux couches peut exister à ce niveau. L'oviducte ne contient pas de plexus intrapariétaux qui assureraient le couplage par voie nerveuse, comme dans le muscle intestinal (Gonella, 1967). On pourrait admettre l'éventualité d'un couplage électrique par conduction musculomusculaire, comme le postule Osa (1974) pour l'utérus de Rate gestante. Un tel mécanisme implique l'existence de faisceaux musculaires de liaison entre les deux couches, dont l'existence n'est pas démontrée. Compte-tenu des délais entre les salves des deux couches, qui peuvent atteindre $3,2 \mathrm{~s}$, on peut aussi avancer l'hypothèse d'un couplage mécanique. L'une des couches en se contractant déformerait ou étirerait l'autre, dont les fibres musculaires répondraient par une dépolarisation et l'apparition de potentiels de pointe.

Reçu en janvier 1982. Accepté en mars 1982.

Remerciements. - Nous remercions le département de Physiologie de I'INRA et I'UER des Sciences Agricoles et Alimentaires de I'Université de Lille I pour leur soutien financier qui a permis la réalisation de ce travail.

\section{Références}

ALM P., ALUMETS J., HAKÄNSON R., OWMAN C., SJÖBERG N. O., SUNDLER F., WALLES B., 1980. Origin and distribution of VIP (Vasoactive Intestinal Peptide) nerves in the genito-urinary tract. Cell Tissue Res., 205, 337-348.

ANAND S., GUHA S. K., 1978. Mechanics of transport of ovum in oviduct. Med. Biol. Eng. Comput., 16, 256-261.

BRUNDIN J., 1965. Distribution and function of adrenergic nerves in the rabbit Fallopian tube. Acta physiol. scand, 66, 5-57. 
BRUNDIN J., FREDRICSSON B., NORBERG K. A., SWEDIN G., 1969. The sympathetic innervation of the oviduct in the rat. Acta physiol. scand., 75, 69-72.

CHALLIS J. R. G., DAVIES I. J., RYAN K. J., 1973. The concentration of progesterone, estrone and estradiol-17 $\beta$ in the plasma of pregnant rabbits. Endocrinology, 93, 971-976.

CHATKOFF M. L., 1975. A biophysicist's view of ovum transport. Gynecol. Invest., 6, 105-122.

COUTINHO E. M., MAIA H. Jr, DE MATTOS C. E. R., 1975. Contractility of the Fallopian tube. Gynecol. Invest., 6, 146-161.

CSAPO A. I., GOODALL M., 1954. Excitability, lenght-tension relation and kinetics of uterine muscle contraction in relation to hormonal status. J. Physiol. London, 126, 384-395.

DANIEL E. E., LUCIEN P., POSEY V. A., PATON D. M., 1975. A functional analysis of the myogenic control systems of the human Fallopian tube. Amer. J. Obstet. Gynecol., 121, 10461053.

GONELLA J., 1967. Etude électrophysiologique de la motricité intestinale. Thèse Doct. Sci. nat., Marseille, $145 \mathrm{p}$.

GONZALEZ DE VARGAS M. I., TALO A., HODGSON B. J., 1976. Correlation between intraluminal pressure of the oviduct and the electrical activity of the longitudinal peritoneal muscle in the rabbit. Biol. Reprod., 15, 492-495.

GREENWALD G. S., 1961. A study of the transport of ova through the rabbit oviduct. Fert. Steril, 12, 80-95.

HALBERT S. A., TAM P. Y., BLANDAU R. J., 1976. Egg transport in rabbit oviduct : the roles of cilia and muscle. Science, 191, 1052-1053.

HARRINGTON F. E., ROTHERMEL J. D., 1977. Daily changes in peripheral plasma progesterone concentrations in pregnant and pseudopregnant rabbits. Life Sci., 20, 1333-1340.

ICHIJO M., 1960. Studies on the motile function of the Fallopian tube. I. Analyse studies on the motile function of the Fallopian tube. Tohoku J. exp. Med., 72, 211-218.

LINDBLOM B., HAMBERGR L., WIQVIST N., 1978. Differentiated contractile effects of prostaglandin- $E$ and prostaglandin- $F$ on isolated circular and longitudinal smooth muscle of human oviduct. Fert. Steril, 30, 553-559.

LINDBLOM B., LJUNG B., HAMBERGER L., 1979. Adrenergic and novel non-adrenergic neuronal mechanisms in the control of smooth muscle activity in the human oviduct. Acta physiol. scand., 106, 215-220.

MEIRI U., MEIRI H., MARSHALL J. M., 1978. Effects of ovarian steroids on spontaneous and nerve-induced electrical activity of the oviduct and its attached membranes in the rabbit. Biol. Reprod., 19, 183-193.

OSA T., 1974. An interaction between the electrical activities of longitudinal and circular smooth muscles of pregnant mouse uterus. Jap. J. Physiol., 24, 189-203.

OWMAN C., SJÖBERG N. O., 1966. Influence of sex hormones on the amount of adrenergic transmitter in the rabbit oviduct, 260-267. In ARRAND KUMAR T. C., Neuroendocrine regulation of fertility. S. Kargel, Basel.

PATON D. M., WIDDICOMBE J. H., RHEAUME D. E., JOHNS A., 1978. The role of adrenergic innervation of the oviduct in the regulation of mammalian ovum transport. Pharmacol. Rev., 29. 67-102.

PAUERSTEIN C. J., 1979. Gamete transport in the mammalian oviduct, 125-138. In BROSENS I., COGNAT M., CONSTANTIN A., THIBIER M., Oviducte et fertilité. Masson, Paris.

RUCKEBUSCH Y., 1975. Relationship between the electrical activity of the oviduct and the uterus of the rabbit in vivo. J. Reprod. Fert., 45, 73-82.

TALO A., 1975. Amplitude variation of the pressure cycles in and between segments of the rabbit oviduct in vitro. Biol. Reprod., 13, 249-254.

TALO A., BRUNDIN J., 1970. Muscular activity in the rabbit oviduct: a combination of electric and mechanic recordings. Biol. Reprod., 5, 67-77.

TALO A., BRUNDIN J., 1973. The functional connections and contractile function of the upper reproductive tract in female rabbits. Biol. Reprod., 9, 142-148.

TALO A., CSAPO A. I., 1970. Conduction of electric activity in late pregnant and parturient rabbit uteri. Physiol. Chem. Phys., 2, 489-494. 
VERDUGo P., LEE W. J., HALBERT S. A., BLANDAU R. J., TAM P. Y., 1980. A stochastic model for oviductal egg transport. Biophys. J., 29. 257-270.

WALLES B., HAKÄNSON R., HELM G., OWMAN C., SJÖBERG N. O., SINDLER F., 1980. Relaxation of human female genital sphincters by the neuropeptide Vasoactive Intestinal Polypeptide. Amer. J. Obstet. Gynecol., 138, 337-338. 